\title{
Exploring fundamental physics with gravitational waves
}

\section{Archil Kobakhidze}

School of Physics, The University of Sydney

E-mail: archil.kobakhidze@sydney.edu.au

The breakthrough discovery of gravitational waves by LIGO and VIRGO Collaborations has opened a new avenue for understanding of some of the most fundamental aspects of the universe. In particular, several well-motivated extensions of the Einstein General Relativity and standard cosmology may now become testable in the strong gravity regime through the measurements of gravitational waves at current and future facilities worldwide. I will give a broad (and somewhat biased) overview of potential implications of those measurements for understanding the quantum nature of black holes, quantum fuzziness of space-time, dark matter and some properties of the Higgs boson and the associated cosmology.

2nd World Summit: Exploring the Dark Side of the Universe 25-29 June, 2018 - EDSU2018

University of Antilles, Pointe-à-Pitre, Guadeloupe, France 


\section{Gravitational waves as a probe of fundamental physics}

The direct observation of gravitational waves from binary black hole mergers [1] represents a triumphal confirmation of Einstein's general covariant theory of gravitation. It has oppened a new era in multimessenger astronomy [2]. In this presentation I would like to argue that current and future observations of gravitational waves also provides with new opportunities for exploring fundamental physical phenomena at microscopic scales, some of which may not be accessible by other means.

The properties of gravitational waves, their production and propagation are largelly defined by symmetries of General Relativity (GR). Deviations from GR and other relevant new physics we are after may potentially manifest in the final gravitational signal through modification in sources and propagation of waves. Taking into account that it took more than 50 years to develop (including important developments in numerical GR [3]) the current state-of-the-art computational tools that allow sufficiently accurate calculations of gravitational waveforms within GR [4], it is not surprising that similar calculations in extended theories of gravity are still in infancy. Therefore, most of the current bounds [5] on new physics coming from the gravitational wave observations are not competitive with the bounds obtained from other astrophysical observations. Lack of proper theoretical modelling of the coalescence regime in almost all relevant modified gravity theories severely limits the true potential of current gravitational wave observations to explore such theories. In the following section I will discuss few interesting recent works in this direction.

\section{Gravitational waves produced by compact binary systems}

The coalescence of a comparable mass binary system proceeds in three phases. The first is the inspiral phase, where the compact objects are well separated with respect to their Schwarzschild radii. The objects are non-relativistic and the inspiral rate is slow relative to the timescale of the orbit. This phase can be treated analytically within the perturbation theory in $v / c$ in for near source waves (Post-Newtonian expansion) and in $G$ for waves far from black holes (Post-Minkowskian expansion). The inspiral phase follows by plunge and merger phase. In this phase, the compact objects are non-relativistic and gravity is strong to rely of perturbative expansion in the Newton's constant $G$. This phase can be treated reliably only using numerical calculations. Finally, there is a ringdown phase, when the highly-distorted remnant formed after merger oscillates, radiating away any deformations and relaxes to a stationary state.

\section{Inspiral phase: probing quantum fuzziness of space-time}

Here I will demonstrate how the observed gravitational wave signals in the inspiral phase put the stringent constraints on the scale of quantum fuzziness of a hypothetical non-commutative space-time [6]. The idea of quantised space-times has first emerged in relation to the short-scale divergences in quantum field theory and traces back to Heisenberg and Pauli. With the advent of the renormalisation program the idea has been forgotten for a while, until in late 90s, when it has been found that quantum space-times emerge in certain field-theoretic limits of the fundametal string theory [7]. The space-time in this scenario exhibits fuzziness characterised by a scale $\ell$, in analogy 
with the quantum fuzziness of the phase space in quantum mechanics, defined through Planck's constant $\hbar$.

The quantum space-time with canonical noncommutativity is defined by a set of operatorvalued space-time coordinates that obey the following commutation relations:

$$
\left[\hat{x}^{\mu}, \hat{x}^{v}\right]=i \ell^{2} \theta^{\mu v}
$$

where $\theta^{\mu v}$ is a real and constant antisymmetric tensor. ${ }^{1}$ A quantum field theory in noncommutative space-time (3.1), is convenient to define in terms of ordinary commutative fields with ordinary products replaced by a nonlocal product (the symmetric Moyal product). Hence noncommutative field theories can be treated (with sufficient care) as an usual field theories with noncommutative corrections $\mathscr{O}\left(\ell^{n}\right)$ at each $n t h$ order term in the Moyal product of fields.

Using field-theoretic methods [8], we were able to compute the energy momentum tensor for binary system including the leading noncommutative correction as [6]:

$$
T^{\mu v}(\mathbf{x}, t)=m_{1} \gamma_{1}(t) v_{1}^{\mu}(t) v_{1}^{v}(t) \delta^{3}\left(\mathbf{x}-\mathbf{y}_{1}(t)\right)+\frac{m_{1}^{3} G^{2} \Lambda^{2}}{8 c^{4}} v_{1}^{\mu}(t) v_{1}^{v}(t) \theta^{k} \theta^{l} \partial_{k} \partial_{l} \delta^{3}\left(\mathbf{x}-\mathbf{y}_{1}(t)\right)+1 \leftrightarrow 2,
$$

where we have simplified the notation by introducing $\Lambda \theta^{i}=\left(\ell M_{P}\right)^{2} \theta^{0 i}$, with $\theta^{i}$ representing the components of a three-dimensional unit vector $\vec{\theta}, \theta^{i} \theta^{i}=1$. In this way $\sqrt{\Lambda}$ parametrise the spacetime noncommutativite scale measured in units of the inverse Planck mass $1 / M_{P}$ (in natural units).

Equipped with the above energy-momentum tensor, we closely follow the formalism of Ref. [4] and after lengthy calculations obtain the noncommutative corrections, which in the leading order appears in the 2PN phase of the gravitational wave signal [6]:

$$
\varphi_{4}=\frac{15293365}{508032}+\frac{27145}{504} v+\frac{3085}{72} v^{2}+\frac{25}{2} \Lambda^{2}(1-2 v) .
$$

This expression reduces to the standard result in the limit $\Lambda \rightarrow 0$, as it should be. Attributing uncertainties in the observe signal, $\left|\delta \varphi_{4}\right| \lesssim 20$ [1], to the above noncommutative correction, we immediately derive the bound [6]:

$$
\sqrt{\Lambda} \lesssim 3.5
$$

This implies that the observed gravitational wave signal constraints the scale of space-time noncommutativity be around the Planck scale. This is 15 orders of magnitude stronger bound than those which follow from particle physics considerations [9].

\section{Merger phase and the black hole soft hair}

In an attempt to resolve the infamous black hole information loss paradox [10], it has been proposed the existence of a nonlocal information transfer accross the black hole horizon through soft modes [11]. It has been also argued that such soft modes may manifest as a deviation from the classical GR picture of evolution of a black hole binary system during the merger phase. To study observable signatures of this proposal, the authors of Ref. [12] modified Einstein's equations by including the soft modes. Their numerical results are somewhat surprising: the soft modes

\footnotetext{
${ }^{1}$ In the string-theoretic setting rhs of Eq. (3.1) represents a background value for an antisymmetric $B$-field.
} 
lead to significant deviations in the observed waveforms not only in the merger phase, but also in the inspiral phase. Although I am not competent to comment on the validity of this particular numerical modelling, it is clear that more work on numerical analysis of the merger phase beyond the GR framework is highly desirable.

\section{Gravitational echoes in the ringdown phase}

Another interesting effect which may potentially be attributed to quantum modification of black hole horizon are gravitational echoes in the ringdown phase []. Consider a compact spherical object of mass $M$ and radius $r_{0}=r_{b h}(1+\varepsilon)$, where $r_{b h}=2 G M$ is the Schwarzschild radius of a black hole of the same mass. Unlike the classical black holes with Schwarzschild horizon, the perturbations of such exotic compact objects (ECOs), such as wormholes, gravastars or black holes with 'quantum horizons', contain trapped modes. For trapped modes of ECOs there are subdominant emissions of gravitational radiation with periodicity $\tau_{\text {echo }} \sim M|\log \varepsilon|$, known as the gravitational echoes. Because of mild $\log \varepsilon$ dependence, the detection of these gravitational echoes can probe distances as close as Planck scale to the horizon, $\varepsilon \sim 1 / M_{P}$ ! In any case, precise measurement of the ringdown phase in future gravitational wave experiments may provide an important information about the nature of black holes/ECOs. Intriguingly, there are some (albeit controversial) claims of detection of such echoes in already existing data [14].

\section{Superradiance and ultralight dark matter}

There is another interesting twist of the direct observation of gravitational waves from black hole binaries. It revived the discussion on primordial black holes serving the role of dark matter in the Universe [15]. On the other hand, black holes themselves in the presence of light scalar dark matter develope superradiant instability and emit gravitational waves [16]. It has been recently shown that the Advanced LIGO detector will be sensitive to detect such gravitational waves for dark matter masses $10^{-14}-10^{-10} \mathrm{eV}$, while the future space based detector LISA will be sensitive to dark matter masses $10^{-19}-10^{-15} \mathrm{eV}$ [17]. It is worth pointing out, that axions $(a)$ and dilatons $(\chi)$ do generically have non-minimal interactions with gravity and electromagnetic field, $a R \tilde{R}$, $a F \tilde{F}, \chi R, \chi F^{2}$. Therefore, one expects modification of gravitational signal also the during black hole coalescence, as well as the electromagnetic follow-up (polarised in the case of axions). These effects need to be considered in complex.

\section{Stochastic gravitational waves from cosmological phase transitions}

It is rather remarkable that through gravitational waves we can 'hear' the Universe from the earliest times, which are not accessible by means of traditional astronomy. Therefore, exploration of gravitational waves from various cosmological sources, such as inflation and phase transitions, may shed light on some of the fundamental questions in particle physics and cosmology. One of particular interest is the properties of the Higgs boson and the nature of the electroweak phase transition. We have argued in Ref. [18], that if the Higgs cubic self-interaction coupling exceeds its Standard Model value by $70 \%$, than the cosmological electroweak phase transition is strongly 
first-order and produces stochastic gravitational waves which may be detected using pulsar timing techniques.

\section{Acknowledgements}

I would like to thank Pierre Petroff and other organisers for the opportunity to attend this stimulating meeting and present the results reported in this paper. I am mostly indebted to my collaborators Cyril Lagger, Adrian Manning and Jason Yue for their contribution. This work was partially supported by the Australian Research Council.

\section{References}

[1] B. P. Abbott et al. [LIGO Scientific and Virgo Collaborations], Phys. Rev. Lett. 116 (2016) 061102.

[2] For an excellent account of the present status and future prospects of gravitational wave astronomy see talk by Laura Cadonati at this conference.

[3] F. Pretorius, Phys. Rev. Lett. 95 (2005) 121101; M. Campanelli, C. O. Lousto, P. Marronetti and Y. Zlochower, Phys. Rev. Lett. 96 (2006) 111101; J. G. Baker, J. Centrella, D. I. Choi, M. Koppitz and J. van Meter, Phys. Rev. Lett. 96 (2006) 111102.

For a review, see,

[4] L. Blanchet, Living Rev. Rel. 17 (2014) 2.

[5] N. Yunes, K. Yagi and F. Pretorius, Phys. Rev. D 94 (2016) 084002.

[6] A. Kobakhidze, C. Lagger and A. Manning, Phys. Rev. D 94 (2016) no.6, 064033 doi:10.1103/PhysRevD.94.064033 [arXiv:1607.03776 [gr-qc]].

[7] N. Seiberg and E. Witten, JHEP 9909 (1999) 032.

[8] A. Kobakhidze, Phys. Rev. D 79 (2009) 047701.

[9] X. Calmet, Eur. Phys. J. C 41 (2005) 269.

[10] S. W. Hawking, Commun. Math. Phys. 43 (1975) 199 Erratum: [Commun. Math. Phys. 46 (1976) 206].

[11] S. B. Giddings, Phys. Rev. D 88 (2013) 064023.

[12] S. L. Liebling, M. Lippert and M. Kavic, JHEP 1803 (2018) 176.

[13] V. Cardoso and P. Pani, Nat. Astron. 1 (2017) 586.

[14] J. Abedi, H. Dykaar and N. Afshordi, Phys. Rev. D 96 (2017) 082004

[15] See the talk by Juan Garcia-Bellido at this conference.

[16] A. Arvanitaki and S. Dubovsky, Phys. Rev. D 83 (2011) 044026.

[17] R. Brito, S. Ghosh, E. Barausse, E. Berti, V. Cardoso, I. Dvorkin, A. Klein and P. Pani, Phys. Rev. Lett. 119 (2017) no.13, 131101.

[18] A. Kobakhidze, C. Lagger, A. Manning and J. Yue, Eur. Phys. J. C 77 (2017) 570. 